\title{
ON THE REFLEXIVITY OF OPERATORS ON FUNCTION SPACES
}

\author{
K. SEDDIGHI AND B. YOUSEFI
}

(Communicated by Paul S. Muhly)

\begin{abstract}
Let $\Omega$ be a bounded plane domain. Sufficient conditions are given so that an operator $T$ in the Cowen-Douglas class $\mathscr{B}_{n}(\Omega)$ is reflexive. The operator $M_{z}$ of multiplication by $z$ on a Hilbert space of functions analytic on a finitely connected domain $\Omega$ is shown to be reflexive whenever $\sigma\left(M_{z}\right)=\bar{\Omega}$ is a spectral set.
\end{abstract}

\section{INTRODUCTION}

For a connected open subset $\Omega$ of the plane and $n$ a positive integer, let $\mathscr{B}_{n}(\Omega)$ be the space introduced by Cowen and Douglas in [3]. This class of operators was further studied by Curto and Salinas [4]. In particular, they show that an operator $T$ in $\mathscr{B}_{n}(\Omega)$ can be realized as the adjoint $T_{\bar{z}}^{*}$ of the operator of multiplication by $\bar{z}$ acting on a Hilbert space $\mathscr{K}_{K}$ of coanalytic functions on $\Omega$ having a generalized Bergman kernel $K$.

In this article we show that if $\Omega$ is a Caratheodory region and $T \in \mathscr{B}_{n}(\Omega)$ is such that $\sigma(T)=\bar{\Omega}$ is a spectral set then $T$ is reflexive. As a result it follows that every contraction in $\mathscr{B}_{n}(\mathbf{D})$, where $\mathbf{D}$ is the open unit disk, is reflexive. Next we show that certain bilateral weighted shifts are reflexive. Finally, we consider a Hilbert space $\mathscr{H}$ of functions analytic on a finitely connected region $\Omega$ that contains the constants and admits multiplication by $z$ as a bounded operator. We show that if $\sigma\left(M_{z}\right)=\bar{\Omega}$ is a spectral set then $M_{z}$ is reflexive.

\section{Preliminaries}

For a connected open subset $\Omega$ of the plane and $n$ a positive integer, let $\mathscr{B}_{n}(\Omega)$ denote the operators $T$ defined on the Hilbert space $\mathscr{H}$ that satisfy

(a) $\Omega \subset \sigma(T)$,

(b) $\operatorname{ran}(T-\omega)=\mathscr{H}$ for $\omega$ in $\Omega$,

(c) $\bigvee_{\omega \in \Omega} \operatorname{ker}(T-\omega)=\mathscr{H}$, and

(d) $\operatorname{dim} \operatorname{ker}(T-\omega)=n$ for $\omega$ in $\Omega$.

Received by the editors November 12, 1990.

1991 Mathematics Subject Classification. Primary 47B37; Secondary 47A25.

Key words and phrases. Reflexive, spectral set, Hilbert space of analytic functions, bilateral weighted shift.

Research parially supported by a grant (no. 69-SC-595-319) from Shiraz University Research Council. 
The space $\mathscr{B}_{n}(\Omega)$ has been introduced by Cowen and Douglas [3] . Now let $\mathscr{H}$ be a separable Hilbert space and let $\mathscr{B}(\mathscr{H})$ denote the algebra of all bounded linear operators on $\mathscr{H}$. Recall that if $A \in \mathscr{B}(\mathscr{H})$, then Lat(A) is by definition the lattice of all invariant subspaces of $A$ and $\operatorname{Alg} \operatorname{Lat}(A)$ is the algebra of all operators $B$ in $\mathscr{B}(\mathscr{H})$ such that $\operatorname{Lat}(A) \subset \operatorname{Lat}(B)$. An operator $A$ in $\mathscr{B}(\mathscr{H})$ is said to be reflexive if $\operatorname{Alg} \operatorname{Lat}(A)=W(A)$, where $W(A)$ is the smallest subalgabra of $\mathscr{B}(\mathscr{H})$ that contains $A$ and the identity $I$ and is closed in the weak operator topology.

In [9] Sarason proved that normal operators are reflexive. It was shown by Deddens [5] that every isometry is reflexive. Olin and Thomson [8] showed that subnormal operators are reflexive. Bercovici, Foias, Langsam, and Pearcy [1] showed that (BCP)-operators are reflexive. The reflexive operators on a finite-dimensional space were characterized by Deddens and Fillmore [6].

It is shown in [4] that every operator in the class $\mathscr{B}_{n}(\Omega)$ is unitarily equivalent to the adjoint of the canonical model associated with a generalized Bergman kernel (g.B.k. for brevity) $K$.

Actually $K$ is the reproducing kernel for a coanalytic functional Hilbert space $\mathscr{K}$ on which we define the operator $T_{\bar{z}}$ of multiplication by $\bar{z}$. The operator $T=T_{\bar{z}}^{*}$ acting on $\mathscr{K}$ is called the canonical model associated with $K$. We know that for every $\lambda$ in $\Omega, T-\lambda$ is onto, $\operatorname{ker}(T-\lambda)=\operatorname{ran} K(\lambda, \cdot)=$ $\left\{K(\lambda, \cdot) \zeta: \zeta \in \mathbf{C}^{n}\right\}$, and $\operatorname{dim} \operatorname{ker}(T-\lambda)=n$. See [4] for a detailed treatment of this subject.

The following characterization of the commutant $\{T\}^{\prime}$ of $T$ is given in Theorem 3.7 of [4], which is stated for the convenience of the reader.

Theorem 1. If $T$ is in $\mathscr{B}_{n}(\Omega)$ and the operator $X$ commutes with $T$, then there exists an analytic function $\Phi: \Omega \rightarrow \mathscr{B}\left(\mathbf{C}^{n}\right)$ such that

$$
X K(\lambda, \cdot)=K(\lambda, \cdot) \Phi(\lambda) \quad(\text { all } \lambda \in \Omega)
$$

and for every $f \in \mathscr{K}_{K}, X^{*} f(\cdot)=(\Phi(\cdot))^{*} f(\cdot)$.

\section{CONDITIONS FOR THE REFLEXIVITY OF THE CANONICAL MODEL}

In this section we give some sufficient conditions on the domain $\Omega$ so that the associated canonical model is reflexive. But first we prove the following

Lemma 1. If $T$ is in $\mathscr{B}_{n}(\Omega)$ then $\operatorname{Alg} \operatorname{Lat}(T) \subset\{T\}^{\prime \prime}$.

Proof. Let $X \in \operatorname{Alg} \operatorname{Lat}(T)$. Each $x \neq 0$ in $\operatorname{ker}(T-z)$ spans a 1-dim invariant subspace of $T$ and hence of $X$. Thus $X$ restricted to $\operatorname{ker}(T-z)$ has the property that every nonzero vector is an eigenvector. So the restriction of $X$ to $\operatorname{ker}(T-z)$ is a scalar multiple of the identity. That is, if $\left\{\gamma_{1}(z), \ldots, \gamma_{n}(z)\right\}$ is a holomorphic spanning set for $\operatorname{ker}(T-z)$, then $X \gamma_{i}(z)=\psi(z) \gamma_{i}(z)$ for some complex number $\psi(z)$, for all $i$ and for each $z$ in $\Omega$.

Fix $z_{0}$ in $\Omega$. Since $X$ is bounded, $X \gamma_{i}(z)$ is holomorphic, so locally $\psi(z)$ can be written as a quotient of holomorphic functions

$$
\psi(z)=\left(\left\langle X \gamma_{i}(z), \gamma_{i}\left(z_{0}\right)\right\rangle\right) /\left(\left\langle\gamma_{i}(z), \gamma_{i}\left(z_{0}\right)\right\rangle\right) .
$$


Finally, $|\psi(z)|\left\|\gamma_{i}(z)\right\|=\left\|X \gamma_{i}(z)\right\| \leq\|X\| \cdot\left\|\gamma_{i}(z)\right\|$ implies $|\psi(z)| \leq\|X\|$, so $\psi$ is in $H^{\infty}(\Omega)$. An application of Theorem 1 shows that $X \in\{T\}^{\prime \prime}$ and the proof of the lemma is complete.

In the next section we show that certain invertible bilateral weighted shifts with adjoint in $\mathscr{B}_{1}(\Omega)$ are reflexive. However, the case of a unilateral weighted shift can easily be taken care of.

Proposition 1. If $T \in \mathscr{B}_{1}(\Omega)$ and $T^{*}$ is an injective unilateral weighted shift, then $T$ is reflexive.

Proof. Theorem 1 of [12] states that $\left\{T^{*}\right\}^{\prime}=W\left(T^{*}\right)$. Thus $\{T\}^{\prime}=W(T)$. By Lemma 1 we have $\operatorname{Alg} \operatorname{Lat}(T) \subset\{T\}^{\prime} \subset W(T)$. Therefore $\operatorname{Alg} \operatorname{Lat}(T)=W(T)$, which proves that $T$ is reflexive.

We now make the following

Definition 1. An open connected subset $U$ of the plane is called a Carathéodory region if its boundary equals the boundary of the unbounded component of $\mathbf{C} \backslash \bar{U}$.

It is easy to see that $U$ is a Carathéodory region if and only if $U$ is the interior of the polynomially convex hull of $\bar{U}$. In this case the Farrell-RubelShields Theorem holds [7, Therem 5.1, p. 151]. Let $f$ be a bounded analytic function on $U$. Then there is a sequence $\left\{p_{n}\right\}$ of polynomials such that $\left\|p_{n}\right\|_{U} \leq C$ for a constant $C$ and $p_{n}(z) \rightarrow f(z)$ for all $z \in U$.

Theorem 2. If $T$ is in $\mathscr{B}_{n}(\Omega)$, where $\Omega$ is a Carathéodory region such that $\sigma(T)=\bar{\Omega}$ is a spectral set for $T$, then $T$ is reflexive.

Proof. Let $K$ be a g.B.k. on $\Omega$ and let $X \in \operatorname{Alg} \operatorname{Lat}(T)$. Then by Lemma 1 $X K(\lambda, \cdot)=\psi(\lambda) K(\lambda, \cdot)$ for some function $\psi \in H^{\infty}(\Omega)$. By the Farrell-RubelShields Theorem, there is a uniformly bounded sequence $\left\{p_{n}\right\}$ of polynomials converging pointwise to $\psi$. Since $\sigma(T)=\bar{\Omega}$ is a spectral set we conclude that $\left\|p_{n}(T)\right\| \leq\left\|p_{n}\right\|_{\Omega} \leq C$ for some constant $C$. By passing to a subsequence we may assume that $p_{n}(T) \rightarrow A$ in the weak operator topology (WOT). Therefore $p_{n}(T) K(\lambda, \cdot) \zeta \rightarrow A K(\lambda, \cdot) \zeta$ weakly. But $p_{n}(T) K(\lambda, \cdot) \zeta=p_{n}(\lambda) K(\lambda, \cdot) \zeta \rightarrow$ $\psi(\lambda) K(\lambda, \cdot) \zeta$ weakly. Hence $A K(\lambda, \cdot) \zeta=\psi(\lambda) K(\lambda, \cdot) \zeta$. From this we conclude that $A=X$, so $X \in W(T)$. Therefore $\operatorname{Alg} \operatorname{Lat}(T) \subset W(T)$ and $T$ is reflexive.

Note that in Theorem 2 we can replace the assumption that $\sigma(T)=\bar{\Omega}$ is a spectral set by the requirement that $\|p(T)\| \leq\|p\|_{\Omega}$ for every polynomial $p$ and the result is still true. In case $T$ is a contraction and $\sigma(T)=\overline{\mathbf{D}}$ von Neumann's inequality holds. That is, $\|p(T)\| \leq\|p\|_{\mathbf{D}}$ for every polynomial $p$. We therefore have the following

Corollary 1. If $T$ is a contraction in $\mathscr{B}_{n}(\mathbf{D})$ then $T$ is reflexive.

\section{Certain bilateral weighted Shifts are Reflexive}

A unilateral (bilateral) weighted shift on a Hilbert space $\mathscr{H}$ is an operator $T$ from $\mathscr{H}$ to itself given by $T e_{n}=w_{n} e_{n+1}$, where $\left\{e_{n}\right\}=\left\{e_{n}\right\}_{n=0}^{\infty}\left(\left\{e_{n}\right\}_{n=-\infty}^{\infty}\right)$ is an orthonormal basis for $\mathscr{H}$ and $\sup _{n}\left|w_{n}\right|<\infty$. 
In his survey article, Shields [11] investigated the properties of weighted shifts. In particular, Question 18 of this article enquires which weighted shifts are reflexive. We shall discuss a class of bilateral shifts that are reflexive.

There is another way of viewing the weighted shifts, which is discussed in [11].

Let $\beta(n)$ be a sequence of positive numbers with $\beta(0)=1$. We consider the space of sequences $f=\{\hat{f}(n)\}$ such that

$$
\|f\|^{2}=\|f\|_{\beta}^{2}=\sum|\hat{f}(n)|^{2}[\beta(n)]^{2}<\infty .
$$

The notation $f(z)=\sum \hat{f}(n) z^{n}$ shall be used whether or not the series converges for any value of $z$. If $n$ ranges over nonnegative integers, these are formal power series; otherwise, they are formal Laurent series. Let $H^{2}(\beta)$ $\left(L^{2}(\beta)\right)$ denote the space of such formal power series (Laurent series).

Let $T$ be an injective unilateral (bilateral) weighted shift operator. It is well known that $T$ can be represented as the operator of multiplication by $z, M_{z}$ on $H^{2}(\beta)\left(L^{2}(\beta)\right)$ for some $\beta$. Let $H^{\infty}(\beta)\left(L^{\infty}(\beta)\right)$ denote the set of formal power series (Laurent series) $\phi(z)=\sum \hat{\phi}(n) z^{n}$ such that $\phi H^{2}(\beta) \subset H^{2}(\beta)$ $\left(\phi L^{2}(\beta) \subset L^{2}(\beta)\right)$.

The relation between $\left\{w_{n}\right\}$ and $\beta$ is $w_{n}=\beta(n+1) / \beta(n)$ (integer $n$ ), $\beta(n)=w_{0} \cdots w_{n-1}(n>0), \beta(0)=1$, and $\beta(-n)=\left(w_{-1} \cdots w_{-n}\right)^{-1}(n>0)$.

If $T$ is an injective bilateral weighted shift with weight sequence $\left\{w_{n}\right\}$ then define

$$
\begin{array}{ll}
r_{1}^{+}(T)=\lim _{n \rightarrow \infty}\left[\inf _{j \geq 0} \beta(n, j)\right]^{1 / n}, & r^{+}(T)=\lim _{n \rightarrow \infty}\left[\sup _{j \geq 0} \beta(n, j)\right]^{1 / n}, \\
r_{1}^{-}(T)=\lim _{n \rightarrow \infty}\left[\inf _{j<0} \beta(-n, j)\right]^{1 / n}, & r^{-}(T)=\lim _{n \rightarrow \infty}\left[\sup _{j<0} \beta(-n, j)^{-1}\right]^{1 / n},
\end{array}
$$

where $\beta(n, j)=\beta(n+j) / \beta(j)$, and

$$
\begin{aligned}
& r_{2}^{+}(T)=\lim -\inf _{n \rightarrow \infty}\left(w_{0} \cdots w_{n-1}\right)^{1 / n}, \\
& r_{3}^{+}(T)=\lim -\sup _{n \rightarrow \infty}\left(w_{0} \cdots w_{n-1}\right)^{1 / n}, \\
& r_{2}^{-}(T)=\lim -\inf _{n \rightarrow \infty}\left(w_{-1} \cdots w_{-n}\right)^{1 / n}, \\
& r_{3}^{-}(T)=\lim -\sup _{n \rightarrow \infty}\left(w_{-1} \cdots w_{-n}\right)^{1 / n},
\end{aligned}
$$

Then $r_{1}^{-} \leq r_{2}^{-} \leq r_{3}^{-} \leq r^{-}$and $r_{1}^{+} \leq r_{2}^{+} \leq r_{3}^{+} \leq r^{+}$. We also let $r(T)$ denote the spectral radius of $T$ and $r_{1}(T)=\lim _{n \rightarrow \infty}\left(m\left(T^{n}\right)\right)^{1 / n}$ where $m(A)=$ $\inf \{\|A x\|:\|x\|=1\}$ for every operator $A$.

Now let $T$ be an injective bilateral weighted shift represented as $M_{z}$ on $L^{2}(\beta)$. Then the commutant $\{T\}^{\prime}$ of $T$ is $L^{\infty}(\beta)$ (where each $\phi \in L^{\infty}(\beta)$ is identified with the operator of multiplication by $\phi, M_{\phi}$ on $\left.L^{2}(\beta)\right)$ [11]. Assume further that $T$ is invertible. If $\left\|T^{-1}\right\|^{-1}=\|T\|$ then $L^{\infty}(\beta)=L^{\infty}(m)$ 
where $m$ is the Lebesgue measure on $|z|=\|T\|\left[11\right.$, p. 80, Theorem $10^{\prime}$ (vii)]. In this case $L^{2}(\beta)=L^{2}(m)$ and $T$ is a scalar multiple of the (unweighted) bilateral shift. Hence $T$ is reflexive.

Assume $\left\|T^{-1}\right\|^{-1}<\|T\|, r_{1}^{-}=r^{-}=\left\|T^{-1}\right\|^{-1}, r_{1}^{+}=r^{+}=\|T\|$, and set $\Omega=\left\{z:\left\|T^{-1}\right\|^{-1}<|z|<\|T\|\right\}$. Then $T^{*} \in \mathscr{B}_{1}(\Omega)$ by [10, Theorem 2.2]. Furthermore $L^{\infty}(\beta)$ coincides, as an algebra with the space of bounded analytic functions in $\Omega$ denoted by $H^{\infty}(\Omega)$. Hence $\{T\}^{\prime}=L^{\infty}(\beta)=H^{\infty}(\Omega)$.

We now show the following

Proposition 2. Let T be an invertible bilateral weighted shift such that $\left\|T^{-1}\right\|^{-1}<$ $\|T\|, r_{1}^{-}=r^{-}=\left\|T^{-1}\right\|^{-1}$ and $r_{1}^{+}=r^{+}=\|T\|$. Then $T$ is reflexive.

Proof. Let $X \in \operatorname{Alg} \operatorname{Lat}(T)$. Then $X \in\{T\}^{\prime}$ by Lemma 1 . Hence $X=M_{\phi}$ for some $\phi \in H^{\infty}(\Omega)$. Since $\phi \in H^{\infty}(\Omega)$, we can write

$$
\phi(z)=\sum_{-\infty}^{\infty} a_{n} z^{n}=\sum_{0}^{\infty} a_{n} z^{n}+\sum_{-\infty}^{-1} a_{n} z^{n}=\phi_{1}(z)+\phi_{2}(z)
$$

where $\phi_{1}$ is bounded and analytic in $|z|<\|T\|$, and $\phi_{2}$ is bounded and analytic in $|z|>\left\|T^{-1}\right\|^{-1}$ [11, Lemma, p. 81]. Since $\phi_{1}$ can be approximated by a sequence of polynomials, we conclude that $M_{\phi_{1}} \in W(T)$. To see this let $\left\{p_{n}\right\}$ be a sequence of polynomials uniformly bounded in $|z|<\|T\|$ converging pointwise to $\phi_{1}$. By von Neumann's inequality

$$
\left\|p_{n}(T)\right\| \leq \sup \left\{\left|p_{n}(z)\right|:|z|<\|T\|\right\} .
$$

Passing to a subsequence, if necessary, we may assume that $p_{n}(T) \rightarrow A$ (WOT) for some operator $A$. Now since every point in $\Omega$ is a bounded point evaluation we conclude that

$$
M_{p_{n}}^{*} k_{w}=\overline{p_{n}(w)} k_{w} \rightarrow \overline{\phi_{1}(w)} k_{w}=M_{\phi_{1}}^{*} k_{w}
$$

for every $w \in \Omega$. On the other hand, $M_{p_{n}}^{*} k_{w} \rightarrow A^{*} k_{w}$ weakly. Therefore, $A^{*} k_{w}=M_{\phi_{1}}^{*} k_{w}$. Since the linear span of $k_{w}, w \in \Omega$, is dense in $L^{2}(\beta)$, we conclude that $M_{\phi_{1}}=A \in W(T)$. We now show that $\phi_{2}=0$. Then $X=M_{\phi}=M_{\phi_{1}} \in W(T)$ and the proof is complete.

To see this note that $H^{2}(\beta)$ is invariant under $T$ so it is invariant under $X$ and $M_{\phi_{1}}$. Hence $\phi_{2}=\phi-\phi_{1}=X 1-M_{\phi_{1}} 1 \in H^{2}(\beta)$. If $\phi_{2} \neq 0$ then $\hat{\phi}_{2}(k) \neq$ 0 for some $k>0$. Since $\phi_{2}$ is bounded and analytic in $|z|>\left\|T^{-1}\right\|^{-1}$, there is a sequence of polynomials $\left\{p_{n}(1 / z)\right\}$ in $1 / z$ uniformly bounded in $|z|>\left\|T^{-1}\right\|^{-1}$ and converging pointwise to $\phi_{2}$. Therefore $p_{n}\left(T^{-1}\right) \rightarrow M_{\phi_{2}}$ (WOT). To see this note that

$$
M_{p_{n}(1 / z)}^{*} k_{w}=\overline{p_{n}(1 / w)} k_{w} \rightarrow \overline{\phi_{2}(w)} k_{w}=M_{\phi_{2}}^{*} k_{w} .
$$


Hence $M_{p_{n}(1 / z)}^{*} f \rightarrow M_{\phi_{2}}^{*} f$ for every $f$ in the linear span of $k_{w}, w \in \Omega$. Now by $\left[11\right.$, Theorem $10^{\prime} \operatorname{vii}($ b), p. 80$]$ we conclude that

$$
\left\|M_{p_{n}(1 / z)}\right\| \leq c \sup \left\{\left|p_{n}(z)\right|:\left\|T^{-1}\right\|^{-1}<|z|<\|T\|\right\}
$$

where $c$ is a constant. Therefore $\left\{\left\|M_{p_{n}(1 / z)}\right\|\right\}$ is uniformly bounded and hence $M_{p_{n}(1 / z)}^{*} f \rightarrow M_{\phi_{2}}^{*} f$ for every $f$ in $L^{2}(\beta)$. We have actually shown that $p_{n}\left(T^{-1}\right) \rightarrow M_{\phi_{2}}$ (SOT). Therefore

$$
\left\langle p_{n}\left(T^{-1}\right) 1, f_{k}\right\rangle \rightarrow\left\langle M_{\phi_{2}} 1, f_{k}\right\rangle
$$

where $f_{k}(z)=z^{k}$. That is, $\hat{p_{n}}(1 / z)(k) \rightarrow \hat{\phi}_{2}(k)$ as $n \rightarrow \infty$. But the left-hand side is zero and the right-hand side is nonzero. This is a contradiction. Hence $\phi_{2}=0$.

The following result of Shields [11, Proposition 15, p. 72] will provide us with a large class of operators satisfying the hypothesis of Proposition 4.1.

Proposition 3. Let $T$ be a bilateral weighted shift with weight sequence $\left\{w_{n}\right\}$. If $w_{n} \rightarrow d^{+}(n \rightarrow \infty)$ and $w_{n} \rightarrow d^{-}(n \rightarrow-\infty)$, then

$$
r_{1}^{-}=\cdots=r^{-}=\left\|T^{-1}\right\|^{-1}=d^{-}, \quad r_{1}^{+}=\cdots=r^{+}=\|T\|=d^{+} .
$$

\section{Finitely CONNECTED DOMAINS}

Suppose $\mathscr{H}$ is a Hilbert space of functions analytic on a plane domain $\Omega$, and suppose that for each point $\lambda \in \Omega$ the linear functional of evaluation at $\lambda$ is bounded on $\mathscr{H}$. We further assume that $\mathscr{H}$ contains the constant functions and multiplication by the independent variable $z$ defines a bounded linear operator $M_{z}$ on $\mathscr{H}$. Note that by the continuity of point evaluations and by the Riesz representation theorem, there exists for each $\lambda \in \Omega$ a unique function $k_{\lambda} \in \mathscr{H}$ such that

$$
f(\lambda)=\left\langle f, k_{\lambda}\right\rangle \quad(f \in \mathscr{H}) .
$$

The function $k_{\lambda}$ is the reproducing kernel for the point $\lambda$, and its norm is the same as that of the corresponding evaluation functional.

Now let $L$ be a compact subset of $\Omega$ and choose $f \in \mathscr{H}$. Then $|f(\lambda)| \leq C_{f}$, $\lambda \in L$, where $C_{f}$ is a constant depending on $f$. This means $\left|\left\langle f, k_{\lambda}\right\rangle\right| \leq C_{f}$, $\lambda \in L$. By the principle of uniform boundedness we have $\left\|k_{\lambda}\right\| \leq C, \lambda \in L$, for some constant $C$. We conclude that convergence in $\mathscr{H}$ implies uniform convergence on compact subsets of $\Omega$.

A complex-valued function $\phi$ on $\Omega$ for which $\phi f \in \mathscr{H}$ for every $f \in \mathscr{H}$ is called a multiplier of $\mathscr{H}$, and the collection of all these multipliers is denoted $\mathscr{M}(\mathscr{H})$. Each multiplier $\phi$ of $\mathscr{H}$ determines a multiplication operator $M_{\phi}$ on $\mathscr{H}$ by the formula $M_{\phi} f=\phi f(f \in \mathscr{H})$. An application of the closed graph theorem shows that $M_{\phi}$ is a bounded operator on $\mathscr{H}$. It is well known that each multiplier is a bounded analytic function on $\Omega$ [12, Lemma 3, p. 782]. 
Actually $\|\phi\|_{\Omega} \leq\left\|M_{\phi}\right\|$. It is also easy to see that if $\phi$ is a multiplier and $\lambda \in \Omega$, then $M_{\phi}^{*} k_{\lambda}=\overline{\phi(\lambda)} k_{\lambda}$.

We now assume that $\Omega$ is a finitely connected domain. It is well known that $\Omega$ is conformally equivalent to a circular domain [2]. By a circular domain we mean any domain that is obtained by removing a finite number of disjoint closed subdisks from the open unit disk. So we let $\Omega=\mathbf{D} \backslash\left(D_{1}^{-} \cup D_{2}^{-} \cup \cdots \cup D_{N}^{-}\right)$ where $D_{i}^{-}=\left\{z:\left|z-z_{i}\right| \leq r_{i}\right\} \quad(i=1, \ldots, N)$ are disjoint closed subdisks of the open unit disk $\mathbf{D}$. We can choose circles $\gamma_{i}=\left\{z:\left|z-z_{i}\right|=r_{i}+\varepsilon_{i}\right\}$ $(i=1, \ldots, N)$ and $\gamma_{0}=\left\{z:|z|=1-\varepsilon_{0}\right\}$ lying in $\Omega$ and concentric to the boundary circles of $\Omega$ so that they do not meet each other. We denote $\Omega_{i}=\mathbf{C} \backslash D_{i}^{-}(i=1, \ldots, N)$.

It is proved in [2] that

$$
H^{\infty}(\Omega)=H^{\infty}(\mathbf{D})+H_{0}^{\infty}\left(\Omega_{1}\right)+\cdots+H_{0}^{\infty}\left(\Omega_{N}\right)
$$

where the subscript zero means that the corresponding functions vanish at $\infty$.

We now prove the following

Theorem 3. Let $\Omega$ be a finitely connected plane domain each point of which is a bounded point evaluation for a Hilbert space $\mathscr{H}$ of functions analytic on $\Omega$ that contains the constant functions and admits multiplication by the independent variable $z, M_{z}$, as a bounded operator. Furthermore, suppose that the map $\phi \rightarrow M_{\phi}$ from $\mathscr{M}(\mathscr{H})$ into $\mathscr{B}(\mathscr{H})$ is an isometry. Then $M_{z}$ is reflexive.

Proof. Set $\mathscr{H}_{0}=\left\{f \in \mathscr{H}: \int_{\gamma_{0}} \zeta^{n} f(\zeta) d \zeta=0, n=0,1,2, \ldots\right\}$. Note that $\mathscr{H}_{0}$ is a subspace of $\mathscr{H}$. To see that $\mathscr{H}_{0}$ is closed let $\left\{f_{k}\right\}$ be a sequence in $\mathscr{H}_{0}$ such that $f_{k} \rightarrow f$ in $\mathscr{H}$. Then $f_{k} \rightarrow f$ uniformly on compact subsets of $\Omega$. It is now easy to see that $f \in \mathscr{H}_{0}$. Clearly $\mathscr{H}_{0}$ is invariant under $M_{z}$ and contains the constants.

We now show that every function in $\mathscr{H}_{0}$ is analytic in $\mathbf{D}$. To see this let $f \in \mathscr{H}_{0}$. Then applying the Cauchy integral formula we can write $f=f_{0}+$ $f_{1}+\cdots+f_{N}$ where $f_{0} \in H(\mathbf{D})$ and $f_{i} \in H_{0}\left(\Omega_{i}\right) \quad(i=1, \ldots, N)$. Set $g=$ $f_{1}+\cdots+f_{N}$. Then $g$ is analytic in $\operatorname{ext}\left(\gamma_{0}^{\prime}\right)$ the unbounded component of $\mathbf{C} \backslash \gamma_{0}^{\prime}$ where the circle $\gamma_{0}^{\prime}$ is chosen sufficiently close to $\gamma_{0}$ with smaller radius so that $\gamma_{0}$ lies in $\operatorname{ext}\left(\gamma_{0}^{\prime}\right)$. We can write

$$
g(z)=\sum_{-\infty}^{-1} a_{n} z^{n}, \quad z \in \operatorname{ext}\left(\gamma_{0}^{\prime}\right) .
$$

Since $f_{0} \in H(\mathbf{D})$, we have $\int_{\gamma_{0}} \zeta^{n} f_{0}(\zeta) d \zeta=0, n=0,1,2, \ldots$; therefore, $\int_{\gamma_{0}} \zeta^{n} g(\zeta) d \zeta=0, n=0,1,2, \ldots$. From this it follows that $g(z)=0$, $z \in \operatorname{ext}\left(\gamma_{0}^{\prime}\right)$. Hence $g \equiv 0$. Therefore $f=f_{0}$ is analytic in $\mathbf{D}$.

Now let $X \in \operatorname{Alg} \operatorname{Lat}\left(M_{z}\right)$. Then by an argument similar to the proof of Lemma 1 we can show that $X=M_{\phi}$ for some multiplier $\phi$. A direct proof is also possible by noting that the one-dimensional span of $\left\{k_{\lambda}\right\}$ is invariant under $M_{z}^{*}$ and hence $X^{*} k_{\lambda}=\overline{\phi(\lambda)} k_{\lambda}$. This too shows that $X=M_{\phi}$. Since $\mathscr{H}_{0}$ is invariant under $M_{z}$, we have $X \mathscr{H}_{0} \subset \mathscr{H}_{0}$, so $X 1=\phi \in \mathscr{H}_{0}$ and hence 
$\phi \in H(\mathbf{D})$. Because $\phi$ is a multiplier, $\phi \in H^{\infty}(\Omega)$. Applying the maximum modulus principle, we conclude that $\phi \in H^{\infty}(\mathbf{D})$ and $\|\phi\|_{\mathbf{D}}=\|\phi\|_{\Omega}$.

Let $\left\{p_{n}\right\}$ be a sequence of polynomials such that $\sup \left\|p_{n}\right\|_{\mathrm{D}}<\infty$ and $p_{n}(z) \rightarrow$ $\phi(z), z \in \mathbf{D}$. Then $\left\|M_{p_{n}}\right\|=\left\|p_{n}\right\|_{\Omega}=\left\|p_{n}\right\|_{\mathbf{D}}<M$ where $M$ is a constant. By passing to a subsequence we may assume that $M_{p_{n}} \rightarrow A$ (WOT). Since $M_{p_{n}}^{*} k_{\lambda}=\overline{p_{n}(\lambda)} k_{\lambda} \rightarrow \overline{\phi(\lambda)} k_{\lambda}, \lambda \in \Omega$, we conclude that $A=X$. Hence $X \in W(T)$. This shows that $T$ is reflexive.

Remark. In Theorem 3 we assumed that the map $\phi \rightarrow M_{\phi}$ from $\mathscr{M}(\mathscr{H})$ into $\mathscr{B}(\mathscr{H})$ is an isometry. We may replace this by the assumption that $\left\|M_{\phi}\right\| \leq$ $c\|\phi\|_{\Omega}$ where $c$ is a constant, or we may suppose that $\sigma\left(M_{z}\right)=\bar{\Omega}$ is a spectral set for $M_{z}$. Even the assumption $\left\|M_{p}\right\|=\|p\|_{\Omega}$ or $\left\|M_{p}\right\| \leq c\|p\|_{\Omega}$ for every polynomial $p$ would be sufficient for our purposes.

Corollary 2. Let $\mathscr{H}$ be a Hilbert space of functions analytic on a finitely connected domain $\Omega$ such that the point evaluations are bounded, $\mathscr{H}$ contains the constants, and $\left\|M_{z}\right\| \leq 1$. Then $M_{z}$ is reflexive.

Proof. By von Neumann's inequality $\left\|M_{p}\right\| \leq\|p\|_{\mathbf{D}}$. But since $\|p\|_{\mathbf{D}}=\|p\|_{\Omega}$ for every polynomial $p$, the conclusion now follows from the Remark.

\section{REFERENCES}

1. H. Bercovici, C. Foias, J. Langsam, and C. Pearcy, $(B C P)$-operators are reflexive, Michigan Math. J. 29 (1982), 371-379.

2. B. Chevereau, C. Pearcy, and A. Shields, Finitely connected domains G, representations of $\mathscr{H}^{\infty}(G)$, and invariant subspaces, J. Operator Theory 6 (1981), 375-405.

3. M. J. Cowen and R. G. Douglas, Complex geometry and operator theory, Acta Math. 141 (1978), 187-261.

4. R. Cutro and N. Salinas, Generalized Bergman kernels and the Cowen-Douglas theory, Amer. J. Math. 106 (1984), 447-488.

5. J. A. Deddens, Every isometry is reflexive, Proc. Amer. Math. Soc. 28 (1971), 509-512.

6. J. A. Deddens and P. A. Fillmore, Reflexive linear transformations, Linear Algebra Appl. 10 (1975), 89-93.

7. T. Gamelin, Uniform algebras, Chelsea, New York, 1984.

8. R. Olin and J. Thomson, Algebras of subnormal operators, J. Funct. Anal. 37 (1980), 271-301.

9. D. Sarason, Invariant subspaces and unstarred operator algebras, Pacific J. Math. 17 (1966), 511-517.

10. K. Seddighi, Essential spectra of operators in the class $\mathscr{B}_{n}(\Omega)$, Proc. Amer. Math. Soc. 87 (1983), 453-458.

11. A. Shields, Weighted shift operators and analytic function theory, Topics in Operator Theory, Math. Surveys Monographs, vol. 13, Amer. Math. Soc., Providence, RI, 1974, pp. 49-128.

12. A. Shields and L. Wallen, The commutants of certain Hilbert space operators, Indiana Univ. Math. J. 20 (1971), 777-788.

Department of Mathematics, Shiraz University, Shiraz, Iran 